\title{
Tratamiento del Diente Permanente Necrótico. Un Cambio de Paradigma en el Campo de la Endodoncia
}

\author{
Treatment of the Necrotic Permanent Tooth. A Paradigm Shift in Endodontics
}

Bucchi, Cristina ${ }^{1}$

BUCCHI, C. Tratamiento del diente permanente necrótico. Un cambio de paradigma en el campo de la endodoncia. Int. J. Odontostomat., 14(4):670-677, 2020.

RESUMEN: El tratamiento de dientes inmaduros necróticos es hoy un gran desafío clínico. La ausencia de cierre del ápice y el reducido grosor de las paredes de la dentina hacen que el tratamiento endodóntico del diente sea difícil e impredecible. Tradicionalmente, estos dientes han sido tratados con apexificación y obturación del canal radicular, sin embargo, con este tratamiento el diente permanece desvitalizado y con paredes de dentina frágiles y cortas, lo que compromete su pronóstico. La endodoncia regenerativa, por el contrario, busca revitalizar el diente e inducir una maduración de la raíz, y se basa en la utilización de las células madre mesenquimales presentes en la región periapical, los factores de crecimiento presentes en la dentina y un andamio que permite el crecimiento de tejido nuevo al interior del canal. Los resultados clínicos son alentadores, ya que en general existe maduración de la raíz y revascularización del diente, sin embargo, el tejido neoformado es tejido de tipo reparativo y, a excepción de estudios ocasionales, no se ha observado regeneración de dentina y pulpa. La endodoncia regenerativa se originó para tratar dientes inmaduros necróticos. Sin embargo, recientemente, estudios preliminares han expandido la aplicación de la endodoncia regenerativa a dientes maduros necróticos, es decir, en pacientes adultos. Los resultados clínicos son positivos y similares a los del diente inmaduro, sin embargo, la investigación referente a la revitalización de dientes maduros se encuentra en etapas tempranas y requiere de un mayor nivel de evidencia antes de ser ofrecida sistemáticamente como terapia a pacientes adultos. Los beneficios potenciales justifican mayor investigación al respecto. Este artículo resume la evidencia científica disponible con respecto a la revitalización de dientes inmaduros y maduros necróticos, sus fundamentos biológicos, los resultados esperados y limitaciones, así como el protocolo clínico.

PALABRAS CLAVE: Endodoncia regenerativa, revitalización, diente inmaduro necrótico, diente maduro necrótico.

\section{INTRODUCCIÓN}

Las terapias médicas en general y odontológicas en particular están cada vez más orientadas a optar por tratamientos conservadores, que aprovechen el potencial reparador y regenerador del cuerpo humano, en lugar de tratamientos de reemplazo del tejido vital perdido por materiales bilógicamente inertes. El descubrimiento de la presencia de células madre en la pulpa dentaria en el año 2000 (Gronthos et al., 2000), y posteriormente en el ligamento periodontal (Seo et al., 2004), la papila apical (Sonoyama et al., 2006), y otros tejidos dentales (Miura et al., 2003), sustentan la posibilidad de regenerar los tejidos dentarios perdidos producto de necrosis pulpar. Estos tratamientos, que son la alternativa terapéutica al tratamiento clásico de apexificación y obturación del canal radicular, son llamados endodoncia regenerativa o revitalización de dientes necróticos maduros e inmaduros, lo que era impensado hace solo algunos años en el campo de la endodoncia.

La primera evidencia de revascularización de dientes depulpados y con lesiones periapicales se publicó en la década de 1960, mediante un estudio en animales de experimentación y en seres humanos (Ostby, 1961). Sin embargo, la investigación sobre este tema no se retomó hasta el año 2001 cuando un reporte de caso de un diente inmaduro necrótico de un paciente de 7 años de edad mostró signos de maduración de raíz, revascularización y curación de

\footnotetext{
${ }^{1}$ Departamento Odontología Integral Adultos, Centro de Investigación en Ciencias Odontológicas, Universidad de La Frontera, Chile.
} 
la lesión periapical, luego de la desinfección del sistema de canales radiculares y un periodo de seguimiento largo (Iwaya et al., 2001). Esta publicación fue seguida de un segundo reporte de caso en 2004, que adicionó la formación intencional de un coagulo de sangre en el interior del canal radicular mediante sobre-instrumentación y que reportó resultados similares a los anteriormente mencionados (Banchs \& Trope, 2004). Estos estudios reactivaron la investigación sobre la revascularización de dientes inmaduros necróticos, que tienen una tasa exponencial de publicación en los últimos 15 años (Kontakiotis et al., 2014, 2015), y un protocolo clínico oficial de la Sociedad Europea de Endodoncia (European Society of Endodontology, 2016) y la Sociedad Americana de Endodoncistas (American Association of Endodontists, 2015). La endodoncia regenerativa o revitalización utiliza las células madre mesenquimales presentes en los tejidos periapicales (Lovelace et al., 2011; Chrepa et al., 2015) y su potencial de diferenciación a células capaces de secretar tejidos mineralizados. Estas células son llevadas al canal radicular mediante la inducción de un coagulo sanguíneo por sobre-instrumentación (Lovelace et al.), y permiten la maduración del diente inmaduro necrótico (Lolato et al., 2016), mejorando su pronóstico. Son numerosos los beneficios de esta terapia, sin embargo, hay aspectos referentes al tipo de tejido formado posterior a la terapía y a la predictibilidad de esta, que continúan sin resolverse, y que serán comentados a profundidad en este artículo.

Como se mencionó, la endodoncia regenerativa se originó para tratar dientes inmaduros necróticos. Sin embargo, recientemente, estudios preliminares han expandido la aplicación de la endodoncia regenerativa a dientes maduros necróticos, es decir, en pacientes adultos (Arslan et al., 2019a,b). Los resultados son alentadores, ya que hay una resolución de la sintomatología clínica, curación de las lesiones periapicales y, en algunos casos, respuesta positiva a test de vitalidad pulpar (Arslan et al., 2019a). La investigación referente a la revitalización de dientes maduros se encuentra en etapas tempranas y requiere de un mayor nivel de evidencia antes de ser ofrecida sistemáticamente como terapia a pacientes adultos, sin embargo, los beneficios potenciales justifican mayor investigación al respecto.

Este artículo resume la evidencia científica disponible con respecto a la revitalización de dientes inmaduros y maduros necróticos, sus fundamentos biológicos, los resultados esperados y limitaciones, así como los protocolos clínicos para cada caso.

\section{Endodoncia regenerativa para dientes inmaduros necróticos}

Bases biológicas. Tradicionalmente los dientes inmaduros necróticos han sido tratados con apexificación que se basa en la aplicación de materiales como el hidróxido de calcio, que induce un cierre apical con tejido reparativo, o MTA, que forma un plug apical (Lin et al., 2017). Ambas técnicas tienen desventajas, como la necesidad de varios recambios y aumento del riesgo de fractura cervical en el caso del hidróxido de calcio (Valera et al., 2015) y dificultad de aplicación, costo económico elevado y riesgo de decoloración de la corona en el caso del MTA (SalemMilani et al., 2017). Más aún, estas técnicas no permiten la maduración de la raíz y el diente permanecerá con paredes cortas y frágiles y desvitalizado permanentemente (Lin et al.), lo que compromete el pronóstico de los dientes tratados.

La endodoncia regenerativa o revitalización se define como una terapia biológica y diseñada para recuperar los tejidos dentales perdidos, incluyendo la dentina y tejidos de la raíz, así como células del complejo dentino-pulpar (Murray et al., 2007). La endodoncia regenerativa sigue los principios de la ingeniería de tejidos, que necesita de tres componentes básicos: células madre con capacidad de diferenciarse al tipo celular deseado, factores de crecimiento que modulen la migración, proliferación y diferenciación de las células madre (Galler et al., 2016) y un andamio que provea un ambiente tridimensional para el crecimiento de tejido nuevo. Aunque se han probado nuevos enfoques añadiendo factores de crecimiento y células crio preservadas (lohara et al., 2011; Zhu et al., 2013) para mejorar los resultados histológicos de la terapia, los tres componentes necesarios para la revitalización de dientes necróticos se encuentran presentes naturalmente en el diente y tejidos periapicales y en consecuencia, la terapia puede ser fácilmente aplicable en la clínica odontológica.

Las células madre son proveídas por los tejidos periapicales (Lovelace et al.,; Chrepa et al., 2015). Se ha comprobado que la papila apical, el tejido adherido al tercio apical de los dientes inmaduros y que es la responsable de la maduración de la raíz en una situación fisiológica, sobrevive a la necrosis pulpar incluso cuando la infección ha comprometido el periápice (Chrepa et al., 2017). Esta estructura contiene las células madre de la papila apical (SCAPs, por sus siglas en inglés), que son llevadas al interior del conducto radicular por medio de sobreinstrumentacion intencio- 
nal del periápice en la endodoncia regenerativa y tienen potencial de diferenciación a células tipo odontoblasto (Lovelace et al.). En un paciente adulto, cuando la papila apical ya no se encuentra presente, las células madre mesenquimales son proveídas por los demás tejidos periapicales (médula ósea, ligamento periodontal y/o sangre) (Chrepa et al., 2015).

Los factores de crecimiento necesarios para la diferenciación de las células madre a células capaces de secretar tejido mineralizado están presentes en la dentina (Galler et al., 2016). Durante el desarrollo embriológico del diente ocurre una interacción epiteliomesénquima, y las células precursoras de odontoblastos y ameloblastos secretan numerosos factores de crecimiento que inducen su diferenciación recíproca, es decir, a odontoblasto y ameloblasto (Goldberg et al., 2011). Estos factores de crecimiento quedan incluidos en la dentina durante toda la vida del paciente y solo son liberados cuando la dentina es demineralizada, ya sea por factores patológicos como la caries o por irrigantes comúnmente utilizados en la odontología como el EDTA (Galler et al., 2016). Por consiguiente, el protocolo clínico de la endodoncia regenerativa incluye la irrigación del canal con EDTA previo a la inducción del coagulo sanguíneo. Los factores de crecimiento liberados por el EDTA son efectivos a nivel de picogramos e inducen la diferenciación de células madre a células tipo odontoblasto (Galler et al., 2011, 2016).

Por último, el andamio es proveído por el propio coágulo sanguíneo que ha invadido el canal radicular posterior a la sobre-instrumentación. Los coágulos sanguíneos se caracterizan por están conformado por una red de fibrina, que sustenta la formación de tejido neoformado. Como alternativa se ha propuesto el uso de fibrina rica en plaquetas (Del Fabbro et al., 2016), esponjas de colágeno y otros, que potencialmente podrían proveer andamios más estables.

Protocolo clínico. Cómo se mencionó anteriormente la aplicación de la endodoncia regenerativa en dientes inmaduros necróticos contó con un alto número de estudios originales en los últimos 15 años (Shamszadeh et al., 2019). Al ser un tratamiento nuevo y no existir directrices estandarizadas, los primeros estudios utilizaron protocolos clínicos que variaban en la medicación e irritantes (Bucchi et al., 2017b), la inducción o no de un coágulo sanguíneo (Bucchi et al., 2017b), la instrumentación o no del canal, los materiales restauradores de la cavidad, así como se incluyeron dientes independiente de la causa de la necrosis pulpar, contribuyendo a producir resultados contradictorios y poco previsibles. La investigación básica, que se fue desarrollando simultáneamente a los estudios clínicos, fue descubriendo que los resultados de terapia se ven influenciados por los factores anteriormente mencionados, y en consecuencia las sociedades de endodoncia europea (ESE) y americana (AAE) elaboraron un protocolo clínico estandarizado y consideraciones clínicas, detallando los materiales que debían ser utilizados. Es probable que el protocolo no sea definitivo y que vaya perfeccionándose, ya que aún es un área de estudio en evolución.

Los factores que alteran los resultados de la endodoncia regenerativa se pueden agrupar en tres: la medicación utilizada durante la terapia, el estado inflamatorio del diente y el periápice y la causa de la necrosis pulpar.

En cuanto a la medicación, se ha comprobado que la clorhexidina al $2 \%$ y el hipoclorito de sodio en concentraciones mayores a $3 \%$, así como las pastas triples antibióticas, son citotóxicas para las células madre y afectan su diferenciación célula tipo odontoblasto y adhesión a la dentina (Galler et al., 2011; Martin et al., 2014; Bucchi et al., 2017b). Por el contrario, el uso de EDTA (Galler et al., 2011, 2015) e hidróxido de calcio (Galler et al., 2015), para la irrigación y medicación de los canales respectivamente, resultan positivos para la adhesión y diferenciación de células madre y son más biocompatibles. La instrumentación debiera no realizarse o limitarse al mínimo para no debilitar las ya frágiles paredes de dentina (European Society of Endodontology).

La infección residual juega un rol fundamental en el fracaso de la terapia, ya que se ha comprobado que cerca del $80 \%$ de los casos de endodoncia regenerativa muestran signos de infección persistente (Almutairi et al., 2019). Estudios in vivo han demostrado que la presencia de bacterias está estadísticamente relacionada con la falta de desarrollo radicular después de la terapia (Verma et al., 2017) y estudios in vitro han demostrado que la presencia de lipopolisacáridos (un componente de la membrana de las bacterias) interfiere con la diferenciación odontogénica de las células madre (Widbiller et al., 2018). Actualmente, la desinfección del diente necrótico recae exclusivamente en el irrigante $(\mathrm{NaOCl})$, cuya concentración está limitada al $3 \%$ para evitar la citotoxicidad sobre las células madre, y en el hidróxido de calcio utilizado como medicación intracanal, ya que no está indicada la instrumentación del diente. Actualmente, se están investigando nuevos enfoques 
que permitan una desinfección eficaz sin alterar negativamente las células madre involucradas y sin debitar la estructura del diente. Uno se estos enfoques es el uso de técnicas físicas como el laser o ultrasonido para potenciar la acción del irrigante, así como el uso de compuestos químicos/biológicos como el propóleo (El-Tayeb et al., 2019), óxido nítrico (Moon et al., 2018) y concentrados de plaquetas autolólogos (Del Fabbro et al.), entre otros. Aunque no existe suficiente evidencia para indicar sistemáticamente el uso de estos compuestos en la terapia, es probable que el protocolo clínico se vea actualizado en un futuro próximo, incorporando alguno de estos $u$ otros factores para mejorar la desinfección de los dientes tratados.

En cuanto a la causa de la necrosis pulpar, algunos estudios señalan que los dientes necrosados debido a traumatismo tienen peores resultados en cuanto a desarrollo radicular que aquellos necrosados por caries o dens evaginatus (Nazzal et al., 2018; Almutairi et al.). La hipótesis que actualmente se expone como causa de esta diferencia es que cuando ocurre un trauma hay un daño de la vaina epitelial de Herwith, estructura que en una situación fisiológica dirige el desarrollo radicular.

El tratamiento de endodoncia regenerativa se realiza en por lo menos tres sesiones (Galler et al., 2016). El número definitivo de sesiones final dependerá de la presencia de sintomatología en los controles posteriores a la aplicación de la medicación intracanal, en cuyo caso deberá repetirse hasta que el diente se encuentre asintomático. La primera sesión se destina a hacer un diagnóstico del caso y a la desinfección del diente. La terapia está contraindicada en dientes con pulpas vitales (en cuyo caso el tratamiento indicado es la apexogénesis), dientes que no se puedan aislar, así como dientes que necesiten una restauración con perno intracraneal posterior (European Society of Endodontology). Si la terapia está indicada para el caso, y posterior a la aislación del diente, se remueve la caries (si aplica) y se retira el tejido necrótico intracanal. La irrigación debe hacerse con $20 \mathrm{ml}$ de $\mathrm{NaOCl}$ entre 1.5 y $3 \%$ (el alto volumen del irrigante mejora su capacidad de desinfección) y suero fisiológico, secando con conos de papel (European Society of Endodontology). La irrigación con sueno no debe omitirse, ya que elimina los restos de $\mathrm{NaOCl}$ del canal. Finalmente, se utiliza hidróxido de calcio como medicación intracanal y se obtura el diente con un material provisional. En la segunda cita, 2 a 4 semanas posterior a la primera, se debe confirmar la ausencia de sintomatología, anestesiar con aneste- sia sin vasocontrictor y aislar el diente. La irrigación con $20 \mathrm{ml}$ de EDTA está indicada para liberar los factores de crecimiento de la dentina y debe hacerse durante 5 minutos (European Society of Endodontology). Posteriormente, se irriga con suero fisiológico y se seca con conos de papel para luego inducir un coagulo de sangre mediante sobreinstrumentación y rotación de la lima. La sangre debiese llegar hasta la unión amelocementaria y se debe esperar a la formación del coagulo, que usualmente tarda 15 minutos. Finalmente se pone una esponja de colágeno sobre el coagulo, cemento silicato hidráulico (ej. MTA) sobre la esponja, composite fluido o vidrio ionómero y finalmente una restauración adhesiva. Idealmente la sangre y el MTA no debieran comprometer la corona del diente, pues podrían decolorarlo. Los controles deben realizarse cada 6 meses hasta completar 2 años de seguimiento (European Society of Endodontology).

\section{Resultados esperados y sus implicaciones}

Estudios clínicos demuestran que la resolución de la sintomatología y curación total o parcial de las lesiones periapicales en casi la totalidad de los dientes tratados (Kontakiotis et al., 2014, 2015; Lin et al.), respuesta positiva a test de vitalidad pulpar en 35-50\% de los casos (Lolato et al.) y desarrollo radicular, en forma de ganancia de grosor, largo y/o cierre apical. El desarrollo de la raíz impacta positivamente en el pronóstico de los dientes, que son menos propensos a fracturarse (Cvek, 1992; Zhou et al., 2017), mientras que la respuesta positiva a test de vitalidad pulpar revela inervación del tejido blando neoformado.

Estudios histológicos en animales muestran que el tejido neoformado no corresponde a pulpa, ya que, si bien es un tejido conectivo laxo y vascularizado, no tiene la organización de la pulpa original y carece de odontoblastos. Por consiguiente, el tejido duro responsable del desarrollo radicular después de la terapia, no es dentina tubular sino un tejido similar al cemento o fibrodentina (Gomes-Filho et al., 2013; Del Fabbro et al.,; Zhou et al.). Por ende, y con los protocolos actuales, no cabe esperar una real regeneración de los tejidos perdidos después de aplicada la terapia. Al día de hoy, solo un reporte de caso en una paciente adolescente ha logrado demostrar regeneración parcial de células tipo odontoblasto y dentina tubular (Austah et al., 2018), sin que se haya logrado identificar la razón de la particularidad de este caso, ya que los medicamentos utilizados y las condiciones de la paciente son los mismas descritos para la vasta mayoría de los casos anteriores, que solo revelan reparación de los tejidos. 


\section{- Nivel de evidencia disponible}

Existen ensayos clínicos (Nagy et al., 2014; Botero et al,. 2017; Jiang et al., 2017; Lin et al.,; Ragab et al., 2019) y meta-análisis (Murray, 2018) que corroboran los resultados positivos de la endodoncia regenerativa y que la sustentan como una terapia de elección para dientes inmaduros necróticos (Lin et al.). Sin embargo, la mayoría de los estudios originales corresponden a reportes o series de caso (Kontakiotis et al., 2014) o estudios en animales (Bucchi et al., 2019a). Adicionalmente, los periodos de seguimiento no suelen ser mayores de 12- 18 meses.

\section{Endodoncia regenerativa para dientes maduros necróticos}

\section{- Bases biológicas}

En principio, las bases biológicas de la endodoncia regenerativa para dientes maduros son las mismas que para los dientes inmaduros. La papila apical es un tejido que desaparece una vez que se ha completado el desarrollo radicular, sin embargo se ha demostrado que la sobreinstrumentación y provocación de coagulo sanguíneo en pacientes adultos trae consigo células mesenquimales provenientes de otros tejidos periapicales (Chrepa et al., 2015). Los demás factores que deben estar presentes en la endodoncia regenerativa, es decir, factores de crecimiento y andamio, no varían con respecto a los del diente inmaduro, ya que como se ha dicho son proveídos por la dentina y el coagulo de sangre, respectivamente. Sin embargo, hay factores que hacen la aplicación de la endodoncia regenerativa un mayor desafío clínico y que deben tomarse en consideración. Estos son el reducido tamaño del foramen apical (Estefan et al., 2016; Bucchi et al., 2017a), que actuaría como barrera física contra el ingreso y formación de tejido intracanal, la complejidad del sistema de canal radicular y por ende de la desinfección, y la menor capacidad regenerativa propia del aumento de edad (Estefan et al.).

\section{- Nivel de evidencia disponible}

El número de artículos publicados sobre endodoncia regenerativa en dientes maduros es considerablemente menor al de dientes inmaduros. Adicionalmente, son artículos más recientes y los periodos de seguimiento son más cortos. Finalmente, los diseños metodológicos se limitan a reportes de caso (Gavino Orduna et al., 2017), estudios en animales (Gomes-Filho et al.), y pocos ensayos clínicos aleatorizados (Arslan et al., 2019a).

\section{- Protocolo clínico}

Al contrario que la endodoncia regenerativa en dientes inmaduros la endodoncia regenerativa en dientes maduros no cuenta con un protocolo estandarizado oficial. Aún así, muchas de las indicaciones de medicamentos e irrigantes para la terapia, tales como el hidróxido de calcio, hipoclorito de sodio al $3 \%$ o menos y el EDTA, son aplicables también a los dientes maduros. Sin embargo, y contrario a los dientes inmaduros, debe hacerse instrumentación del canal para ampliar el tamaño del foramen apical y así facilitar el ingreso y crecimiento de tejido nuevo (Estefan et al.), facilitar el ingreso del irrigante a los canales accesorios y también reducir la cantidad de dentina infectada. Al día de hoy, no existe consenso sobre el diámetro final que debiese tener el foramen apical para favorecer en los resultados de la terapia (He et al., 2017). Algunos estudios sugieren un tamaño del foramen apical mínimo de 0,6 a $1 \mathrm{~mm}$ (Saoud et al., 2014; Bucchi et al., 2019a).

\section{Resultados esperados}

Al igual que en dientes inmaduros, los estudios evidencian resolución de la sintomatología y lesiones periapicales (Arslan et al., 2019a), y formación de tejido conectivo laxo vascularizado y ausencia de odontoblastos (Arslan et al., 2019b; Bucchi et al., 2019a). También se reporta respuesta positiva a test de vitalidad pulpar en aproximadamente el $50 \%$ de los casos (Arslan et al., 2019a). Aunque no es un resultado buscado, pues la raíz ya ha completado su desarrollo radicular, existe evidencia de formación de tejido mineralizado adherido al foramen apical ampliado y al canal radicular (Bucchi et al., 2019a).

\section{Problemas derivados de la falta de regeneración}

Diferencias estructurales y funcionales entre los odontoblastos y las células presentes después de la revitalización (fibroblastos-osteoblastos y otros tipos de células), y entre la dentina tubular y los tejidos reparativos tienen una importancia clínica crítica. La formación de células similares a los odontoblastos es necesaria para restablecer las funciones de la pulpa, como la respuesta inmune, la secreción de dentina y la percepción del dolor y estímulos mecánicos (Couve et al., 2013; Farges et al., 2009, 2015). 
Para realizar estas funciones propias de los odontoblastos, las células diferenciadas después de las terapias deben presentar los receptores adecuados (Farges et al., 2011) y presentar una extensión celular lo más coronalmente posible en una estructura tubular para detectar lesiones tempranas, desencadenar una respuesta inmunitaria y secretar de dentina terciaria (Couve et al., 2014). Sin embargo, los tejidos reparativos (tejido similar al cemento o fibrodentina) que se producen después de las terapias son, a diferencia de la dentina, estructuras atubulares (Nosrat et al., 2015). La falta de procesos celulares en el tejido duro neoformado compromete las funciones inmunológicas y sensoriales de la pulpa, así como la secreción de dentina terciaria. La incapacidad del diente para reaccionar a una nueva lesión de caries compromete el pronóstico del diente.

Adicionalmente, el cemento dental tiene una menor dureza y elasticidad que la dentina (Ho et al., 2010). Por lo tanto, la formación de tejido similar al cemento en lugar de la dentina tiene un impacto en el rendimiento biomecánico del diente al soportar fuerzas físicas (Bucchi et al., 2019b), como masticación, traumatismos dentoalveolares o movimientos de ortodoncia, ya que los dientes reforzados con cemento distribuyen la tensión mecánica de manera desventajosa en comparación con los dientes reforzados con dentina (Bucchi et al., 2019b). La fortaleza ante la tensión mecánica es un requisito previo para la supervivencia dental a largo plazo y, por lo tanto, la formación de tejido reparador puede hacer al diente más propenso a fracturas.

BUCCHI, C. Treatment of the necrotic permanent tooth. A paradigm shift in endodontics. Int. J. Odontostomat., 14(4): 670-677, 2020.

ABSTRACT. Nowadays, the treatment of immature necrotic teeth is an important clinical challenge. The absence of apex closure and low thickness of the dentin walls, make endodontic treatment unpredictable and difficult. Traditionally, these teeth have been treated with apexification and obturation of the root canal. As a result of this treatment, the tooth remains devitalized and with fragile and short dentin walls, which compromises its prognosis. Regenerative endodontics, on the other hand, seeks to revitalize the tooth and induce root maturation, and is based on the use of mesenchymal stem cells present in the periapical tissues, growth factors present in the dentin and a scaffold that allows growth of new tissue in the root canal. The clinical results are encouraging, since generally, there is root maturation and revascularization of the tooth. However, the newly formed tissue is reparative tissue and with the exception of some studies, no regeneration of dentin and pulp has been reported. Regenerative endodontics emerged to treat necrotic immature teeth. However, recently, preliminary studies have applied regenerative endodontics in mature necrotic teeth, in adult patients. Preliminary results are positive and are similar to those of immature teeth. Nevertheless, research regarding the revitalization of mature teeth is in the early stages and requires further evidence before being systematically administered as therapy in adult patients. However, the potential benefits justify further research in this regard. This article summarizes the available scientific evidence regarding the revitalization of immature and mature necrotic teeth, their biological basis, the expected results and limitations, as well as the clinical protocols for each case.

KEY WORDS: Regenerative endodontics, revitalization, immature necrotic tooth, mature necrotic tooth.

\section{REFERENCIAS BIBLIOGRÁFICAS}

American Association of Endodontists (AAE). Clinical Considerations for a Regenerative Procedure. Chicago, American Association of Endodontists, 2015.

Almutairi, W.; Yassen, G. H.; Aminoshariae, A.; Williams, K. A. \& Mickel, A. Regenerative endodontics: a systematic analysis of the failed cases. J. Endod., 45(5):567-77, 2019.

Arslan, H.; Ahmed, H. M. A.; Sahin, Y.; Yildiz, E. D.; E, Gündog ¿du, E. C.; Guven, Y. \& Khalilov, R. Regenerative endodontic procedures in necrotic mature teeth with periapical radiolucencies: a preliminary randomized clinical study. J. Endod., 45(7):863-72, 2019a.

Arslan, H.; Sahin, Y.; Topcuoglu, H. S. \& Gundogdu, B. Histologic evaluation of regenerated tissues in the pulp spaces of teeth with mature roots at the time of the regenerative endodontic procedures. J. Endod., 45(11):1384-9, 2019b.

Austah, O.; Joon, R.; Fath, W. M.; Chrepa, V.; Diogenes, A.; Ezeldeen, M.; Couve, E. \& Ruparel, N. B. Comprehensive Characterization of 2 Immature Teeth Treated with Regenerative Endodontic Procedures. J. Endod., 44(12):1802-11, 2018.

Banchs, F \& Trope, M. Revascularization of immature permanent teeth with apical Periodontitis: New Treatment protocol? J. Endod., 30(4):196-200, 2004.

Botero, T. M.; Tang, X.; Gardner, R.; Hu, J. C. C.; Boynton, J. R. \& Holland, G. R. Clinical evidence for regenerative endodontic procedures: immediate versus delayed induction? J. Endod., 43(9S):S75-81, 2017.

Bucchi, C.; Gimeno-Sandig, A. \& Manzanares-Céspedes, C. Enlargement of the apical foramen of mature teeth by instrumentation and apicoectomy. A study of effectiveness and the formation of dentinal cracks. Acta Odontol. Scand., 75(7):488-95; 2017a.

Bucchi, C.; Gimeno-Sandig, Á.; Valdivia-Gandur, I.; ManzanaresCéspedes, C. \& de Anta, J. M. A Regenerative endodontic approach in mature ferret teeth using rodent preameloblastconditioned medium. In Vivo (Brooklyn), 33(4):1143-50, 2019a.

Bucchi, C.; Marcé-Nogué, J.; Galler, K. M. \& Widbiller, M. Biomechanical performance of an immature maxillary central incisor after revitalization: A finite element analysis. Int. Endod. J., 52(10):1508-18, 2019b. 
Bucchi, C.; Valdivia-Gandur, I.; Sánchez-Bizjak, R.; Tallón-Walton, V. \& Manzanares-Céspedes, C. Regenerative endodontic therapy: A systematic review of clinical protocols. Int. J. Clin. Exp. Med., 10(2):2006-15, 2017b.

Chrepa, V.; Henry, M. A.; Daniel, B. J. \& Diogenes, A. Delivery of Apical Mesenchymal Stem Cells into Root Canals of Mature Teeth. J. Dent. Res., 94(12):1653-9; 2015.

Chrepa, V.; Pitcher, B.; Henry, M. A. \& Diogenes, A. Survival of the apical papilla and its resident stem cells in a case of advanced pulpal necrosis and apical periodontitis. J. Endod., 43(4):5617, 2017.

Couve, E.; Osorio, R. \& Schmachtenberg, O. Reactionary dentinogenesis and neuroimmune response in dental caries. $J$. Dent. Res., 93(8):788-93, 2014.

Couve, E.; Osorio, R. \& Schmachtenberg, O. The amazing odontoblast: Activity, autophagy, and aging. J. Dent. Res., 92(9):765-72, 2013

Cvek, M. Prognosis of luxated non-vital maxillary incisors treated with calcium hydroxide and filled with gutta-percha. A retrospective clinical study. Endodontics \& Dental Traumatology. Endod. Dent. Traumatol., 8:45-55, 1992.

Del Fabbro, M.; Lolato, A.; Bucchi, C.; Taschieri, S. \& Weinstein, R. L. Autologous platelet concentrates for pulp and dentin regeneration: a literature review of animal studies. J. Endod., 42(2):250-7, 2016.

El-Tayeb, M. M.; Abu-Seida, A. M.; El Ashry, S. H. \& El-Hady, S. A. Evaluation of antibacterial activity of propolis on regenerative potential of necrotic immature permanent teeth in dogs. BMC Oral Health, 19(1):1-12, 2019.

Estefan, B. S.; El Batouty, K. M.; Nagy, M. M. \& Diogenes, A. Influence of age and apical diameter on the success of endodontic regeneration procedures. J. Endod., 42(11):1620-5, 2016.

European Society of Endodontology; Galler, K. M.; Krastl, G.; Simon, S.; Van Gorp, G.; Meschi, N.; Vahedi, B. \& Lambrechts, P. European Society of Endodontology position statement: Revitalization procedures. Int. Endod. J., 49(8):717-23, 2016.

Farges, J. C.; Bellanger, A.; Ducret, M.; Aubert-Foucher, E.; Richard, B.; Alliot-Licht, B.; Bleicher, F. \& Carrouel, F. Human odontoblastlike cells produce nitric oxide with antibacterial activity upon TLR2 activation. Front. Physiol., 6:185, 2015.

Farges, J. C.; Carrouel, F.; Keller, J. F.; Baudouin, C.; Msika, P.; Bleicher, F. \& Staquet, M. J. Cytokine production by human odontoblast-like cells upon Toll-like receptor-2 engagement. Immunobiology, 216(4):513-7, 2011.

Farges, J. C.; Keller, J. F.; Carrouel, F.; Durand, S. H.; Romeas, A.; Bleicher, F.; Lebecque, S. \& Staquet, M. J. Odontoblasts in the dental pulp immune response. J. Exp. Zool. Part B Mol. Dev. Evol., 312(5):425-36, 2009.

Galler, K. M.; Buchalla, W.; Hiller, K. A.; Federlin, M.; Eidt, A.; Schiefersteiner, M. \& Schmalz, G. Influence of root canal disinfectants on growth factor release from dentin. J. Endod., 41(3):363-8, 2015.

Galler, K. M.; D'Souza, R. N.; Federlin, M.; Cavender, A. C.; Hartgerink, J. D.; Hecker, S. \& Schmalz, G. Dentin conditioning codetermines cell fate in regenerative endodontics. J. Endod., 37(11):1536-41, 2011.

Galler, K. M.; Widbiller, M.; Buchalla, W.; Eidt, A.; Hiller, K. A.; Hoffer, P. C. \& Schmalz, G. EDTA conditioning of dentine promotes adhesion, migration and differentiation of dental pulp stem cells. Int. Endod. J., 49(6):581-90, 2016.

Gavino Orduna, J. F.; Caviedes-Bucheli, J.; Manzanares-Cespedes, M. C.; Berastegui-Jimeno, E.; Martin Biedma, B.; Segura-Egea, J. J. \& López-López, J. Use of platelet-rich plasma in endodontic procedures in adults: regeneration or repair? A report of 3 cases with 5 years of follow-up. J. Endod., 43(8):1294-301, 2017.
Goldberg, M.; Kulkarni, A.; Young, M. \& Boskey A. Dentin: structure, composition and mineralization. Front Biosci. (Elite Ed.), 3:71135, 2011.

Gomes-Filho, J. E.; Tobias Duarte, P. C.; Ervolino, E.; Mogami Bomfim, S. R.; Xavier Abimussi, C. J.; Mota Da Silva Santos, L.; Lodi, C. S.; De Oliveira, S. H. P.; Dezan Jr., E. \& Cintra, L. T. A. Histologic characterization of engineered tissues in the canal space of closed-apex teeth with apical periodontitis. $J$. Endod., 39(12):1549-56, 2013.

Gronthos, S.; Mankani, M.; Brahim, J.; Robey, P. G. \& Shi, S. Postnatal human dental pulp stem cells (DPSCs) in vitro and in vivo. Proc. Natl. Acad. Sci. U. S. A., 97(25):13625-30, 2000

He, L.; Kim, S. G.; Gong, Q.; Zhong, J.; Wang, S.; Zhou, X.; Ye, L.; Ling, J. \& Mao, J. J. Regenerative Endodontics for Adult Patients. J. Endod., 43(9S):S57-S64, 2017.

Ho, S. P.; Yu, B.; Yun, W.; Marshall, G. W.; Ryder, M. I. \& Sally, J. Structure, chemical composition and mechanical properties of human and rat cementum and its interface with root dentin. Acta Biomater., 5(2):707-18, 2010.

Iohara, K.; Imabayashi, K.; Ishizaka, R.; Watanabe, A.; Nabekura, J.; Ito, M.; Matsushita, K.; Nakamura, H. \& Nakashima, M. Complete pulp regeneration after pulpectomy by transplantation of CD105+ stem cells with stromal cell-derived factor-1. Tissue Eng. Part A, 17(15-16):1911-20, 2011.

Iwaya, S. I.; Ikawa, M. \& Kubota, M. Revascularization of an immature permanent tooth with apical periodontitis and sinus tract. Dent. Traumatol., 17(4):185-7, 2001.

Jiang, X.; Liu, H. \& Peng, C. Clinical and radiographic assessment of the efficacy of a collagen membrane in regenerative endodontics: a randomized, controlled clinical trial. J. Endod., 43(9):1465-71, 2017.

Kontakiotis, E. G.; Filippatos, C. G. \& Agrafioti, A. Levels of evidence for the outcome of regenerative endodontic therapy. J. Endod., 40(8):1045-53, 2014.

Kontakiotis, E. G.; Filippatos, C. G.; Tzanetakis, G. N. \& Agrafioti, A. Regenerative endodontic therapy: A data analysis of clinical protocols. J. Endod., 41(2):146-54, 2015.

Lin, J.; Zeng, Q.; Wie, X.; Zhao, W.; Cui, M.; Gu, J.; Lu, J.; Yang, M. \& Ling, J. Regenerative endodontics versus apexification in immature permanent teeth with apical periodontitis: a prospective randomized controlled study. J. Endod., 43(11):1821-7, 2017.

Lolato, A.; Bucchi, C.; Taschieri, S.; Kabbaney, A. E. \& Fabbro, M. D. Platelet concentrates for revitalization of immature necrotic teeth: a systematic review of the clinical studies. Platelets, 27(5):383-92, 2016

Lovelace, T. W.; Henry, M. A.; Hargreaves, K. M. \& Diogenes, A. Evaluation of the delivery of mesenchymal stem cells into the root canal space of necrotic immature teeth after clinical regenerative endodontic procedure. J. Endod., 37(2):133-8, 2011.

Martin, D. E.; De Almeida, J. F. A.; Henry, M. A.; Khaing, Z. Z.; Schmidt, C. E.; Teixeira, F. B. \& Diogenes, A. Concentrationdependent effect of sodium hypochlorite on stem cells of apical papilla survival and differentiation. J. Endod., 40(1):51-5, 2014.

Miura, M.; Gronthos, S.; Zhao, M.; Lu, B.; Fisher, L. W.; Robey, P. G. \& Shi, S. SHED: stem cells from human exfoliated deciduous teeth. Proc. Natl. Acad. Sci. U. S. A., 100(10):5807-12, 2003.

Moon, C. Y.; Nam, O. H.; Kim, M.; Lee, H. S.; Kaushik, S. N.; Walma, D. A. C.; Jun, H. W.; Cheon, K. \& Choi, S. C. Effects of the nitric oxide releasing biomimetic nanomatrix gel on pulp-dentin regeneration: Pilot study. PLoS One. 13(10):e0205534, 2018.

Murray, P. E. Platelet-rich plasma and platelet-rich fibrin can induce apical closure more frequently than blood-clot revascularization for the regeneration of immature permanent teeth: a meta-analysis of clinical efficacy. Front. Bioeng. Biotechnol., 6:139, 2018. 
Murray, P. E.; Garcia-Godoy, F. \& Hargreaves, K. M. Regenerative endodontics: a review of current status and a call for action. $J$. Endod., 33(4):377-90, 2007.

Nagy, M. M.; Tawfik, H. E.; Hashem, A. A. R. \& Abu-Seida, A. M. Regenerative potential of immature permanent teeth with necrotic pulps after different regenerative protocols. J. Endod., 40(2):192-8, 2014.

Nazzal, H.; Kenny, K.; Altimimi, A.; Kang, J. \& Duggal, M. S. A prospective clinical study of regenerative endodontic treatment of traumatized immature teeth with necrotic pulps using biantibiotic paste. Int. Endod. J., 51:e204-15, 2018.

Nosrat, A.; Kolahdouzan, A.; Hosseini, F.; Mehrizi, E. A.; Verma, P. \& Torabinejad, M. Histologic outcomes of uninfected human immature teeth treated with regenerative endodontics: 2 case reports. J. Endod., 41(10):1725-9, 2015.

Ostby, B. N. The role of the blood clot in endodontic therapy. An experimental histologic study. Acta Odontol. Scand., 19:32453, 1961.

Ragab, R. A.; Lattif, A. E. A. E. \& Dokky, N. A. E. W. E. Comparative study between revitalization of necrotic immature permanent anterior teeth with and without platelet rich fibrin: a randomized controlled trial. J. Clin. Pediatr. Dent., 43(2):78-85, 2019.

Salem-Milani, A.; Ghasemi, S.; Rahimi, S.; Ardalan-Abdollahi, A. \& Asghari-Jafarabadi, M. The discoloration effect of white mineral trioxide aggregate (WMTA); calcium enriched mixture (CEM), and portland cement (PC) on human teeth. J. Clin. Exp. Dent., 9(12):e1397-401, 2017.

Saoud, T. M.; Sigurdsson, A.; Rosenberg, P. A.; Lin, L. M. \& Ricucci, D. Treatment of a large cystlike inflammatory periapical lesion associated with mature necrotic teeth using regenerative endodontic therapy. J. Endod., 40(12):2081-6, 2014.

Seo, B. M.; Miura, M.; Gronthos, S.; Bartold, P. M.; Batouli, S.; Brahim, J.; Young, M.; Robey, P. G.; Wang, C. Y. \& Shi, S. Investigation of multipotent postnatal stem cells from human periodontal ligament. Lancet, 364(9429):149-55, 2004.

Shamszadeh, S.; Asgary, S. \& Nosrat, A. Regenerative endodontics: a scientometric and bibliometric analysis. J. Endod., 45(3):27280, 2019.

Sonoyama, W.; Liu, Y.; Fang, D.; Yamaza, T.; Seo, B.; Zhang, C.; Liu, H.; Gronthos, S.; Wang, C. Y.; Wang, S. \& Shi, S. Mesenchymal stem cell-mediated functional tooth regeneration in swine mesenchymal stem cell-mediated functional tooth regeneration in swine. PLoS One, 1(1):e79, 2006.

Valera, M. C.; Albuquerque, M. T. P.; Yamasaki, M. C.; Vassallo, F. N. S.; da Silva, D. A. E. S. A. \& Nagata, J. Y. Fracture resistance of weakened bovine teeth after long-term use of calcium hydroxide. Dent. Traumatol.. 31(5):385-9, 2015.

Verma, P.; Nosrat, A.; Kim, J. R.; Price, J. B.; Wang, P.; Bair, E.; Xu, H. H. \& Fouad, A. F. Effect of residual bacteria on the outcome of pulp regeneration in vivo. J. Dent. Res., 96(1):100-6, 2017.

Widbiller, M.; Eidt, A.; Wolflick, M.; Lindner, S. R.; Schweikl, H.; Hiller, K. A.; Buchalla, W. \& Galler, K. M. Interactive effects of LPS and dentine matrix proteins on human dental pulp stem cells. Int. Endod. J., 51(8):877-88, 2018.

Zhou, R.; Wang, Y.; Chen, Y.; Chen, S.; Lyu, H.; Cai, Z. \& Huang, X. Radiographic, histologic, and biomechanical evaluation of combined application of platelet-rich fibrin with blood clot in regenerative endodontics. J. Endod., 43(12):2034-40, 2017.

Zhu, W.; Zhu, X.; Huang, G. T. J.; Cheung, G. S. P.; Dissanayaka, W. L. \& Zhang, C. Regeneration of dental pulp tissue in immature teeth with apical periodontitis using platelet-rich plasma and dental pulp cells. Int. Endod. J., 46(10):962-70, 2013.
Dirección para correspondencia:

Prof. Dr. Cristina Bucchi

cristina.bucchi@ufrontera.cl

Facultad de Odontología

Av. Francisco Salazar 01145

Temuco

CHILE

Email: cristina.bucchi@ufrontera.cl

Recibido : 13-04-2020

Aceptado: 24-06-2020 